\title{
MODULATED STRUCTURES OF ORDER PARAMETER AND SPONTANEOUS PARITY BREAKING
}

\author{
V.F.KLEPIKOV, S.V.BEREZOVSKY \\ Scientific and Technical Centre for Electrophysical Treatment \\ National Ukrainian Academy of Science \\ 3 Valtera St., a/b 9410, UA-310108 Kharkiv, Ukraine
}

Received September 12, 1995

\begin{abstract}
Modulated structures of order parameter are considered as an important necessary element of phase transitions. Stable modulated structures are a result of the competition among several high gradient terms. There are two phases of modulated structures which have different level of spontaneous symmetry breaking for the parity group. At the transition point between these phases the space distribution of the order parameter is similar to that of bell-soliton. Thermodynamic potential is evaluated in the vicinity of this point.
\end{abstract}

Critical phenomena near a phase transition (PT) point are usually described as nonequilibrium ones. But there are some important reasons, first of all energetic and symmetrical, to consider critical inhomogeneities of the order parameter (OP) as the equilibrium extremals of a thermodynamical potential. Modulated OP structures are just an example of such phenomena [1]. Modulated structures (MS) are energetically advantageous (unlike domain structures) even in an unbounded media due to the competition among gradient terms in the system energy expression. Lots of physical systems may be described by a scalar one-component OP (ferroelectrics, some magnetics, etc.). In this case the following universal expression for the thermodynamical potential $\Phi$ is used [1,2]:

$$
\Phi=\Phi_{0} \int\left[A_{0}\left(\phi^{\prime \prime}\right)^{2}-g\left(\phi \phi^{\prime}\right)^{2}-\gamma\left(\phi^{\prime}\right)^{2}+r \phi^{2}+\frac{s}{2} \phi^{4}+\frac{1}{3} B_{0} \phi^{6}\right] d x,
$$

where $\phi(x)$ is the scalar OP field; $\phi^{\prime}(x)=\frac{\partial \phi(x)}{\partial x}, A_{0}, g, \gamma, r, s, B_{0}$ are material constants. It should be emphasized that the stable $\phi(x)$ structure can arise only as a result of the competition among several different gradient terms (or from specific boundary conditions, as in the case of domain structures).

Euler-Poisson variational differential equation corresponding to (1) has the form:

$$
A_{0} \phi^{(I V)}+g\left(\phi^{2} \phi^{\prime \prime}+\phi \phi^{2}\right)+\gamma \phi^{\prime \prime}+r \phi+s \phi^{3}+B_{0} \phi^{5}=0 .
$$

Using the substitution $w(z)=\left(\phi^{\prime}\right)^{2}, z=\phi^{2}(x)$ the first integral of equation (2) may be written as the 2 nd-order differential nonlinear equation

$$
2 A_{0}\left(w w_{z}^{\prime}+2 z w w_{z}^{\prime \prime}\right)-A_{0} z\left(w_{z}^{\prime}\right)^{2}+(\gamma+g z) w+r z+\frac{s}{2} z^{2}+\frac{1}{3} B_{0} z^{3}=D,
$$

(C) V.F.Klepikov, S.V.Berezovsky, 1996

ISSN 0452-9910. Condensed Matter Physics 1996 No 8 (69-74) 
where $D$ is the integration constant. Keeping in mind that $z=\phi^{2}$ is small, let us assume that $w(z)=\sum_{n=0}^{\infty} a_{n} z^{n}$, in other words, $\phi^{2}(x)=\sum_{n=0}^{\infty} a_{n} \phi^{2 n}$. There are three specific cases of this solution:

1). $a_{0} \neq 0 ., a_{1} \neq 0 .-\phi(x)$ may be a periodic function;

2). $a_{0}=0 ., a_{1} \neq 0 .-\phi(x)$ has an exponential asymptotics, i.e. $\phi(x) \approx F\{\exp (-x)\} ; x \rightarrow \infty$;

3). $a_{0}=0 ., a_{1}=0 ., a_{2} \neq 0 .-\phi(x) \approx F\left\{x^{-1}\right\} ; x \rightarrow \infty$.

It should be noted that in the case 3 ) the material parameter $r$ has to be equal to zero. The situations when $a_{0}=0 ., a_{1}=0 ., a_{2}=0$. and $a_{3} \neq 0$. and so on are similar to the third case. Therefore for qualitative analysis we can use the truncated series

$$
\phi^{\prime 2}(x)=a_{0}+a_{1} \phi^{2}+a_{2} \phi^{4} .
$$

Now let us classify the phases originating in the model (1), (3) and their symmetries.

1. $\phi(x)=0 .=$ const. There is no MS in the system (figure 1). Symmetry with respect to the parity group $(\phi \rightarrow-\phi)$ and translational group $(x \rightarrow$ $x+C)$ takes place.
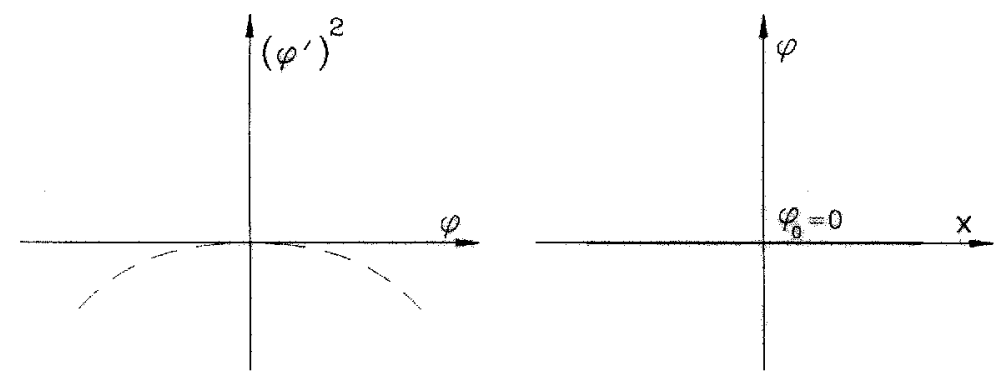

Figure 1.

2. The growth of $\phi(x)$ fluctuations and the instability of $\phi(x)=0$ state at $r=\gamma^{2} / 4 A_{0}$ lead to the 2nd-kind PT into the phase MS1. Owing to the fact that $\phi(x) \approx 0$ this PT may be described as a "linear" one, in the linear approximation of (2). For the model (3) the solution to equation (2) is $\phi(x)=a_{c} c n\left(b_{c} x, k\right)$, where $c n(x, k)$ is the Jacobi elliptic function, the parameters $a_{c}, b_{c}, k$ depend on the material constants. So $\phi(x)$ is a periodic function with the period $T=K(k)$, where $K(k)$ is the normal 1st-kind elliptic integral. For small values of $k$ the space distribution of $\phi(x)$ may be approached with

$$
\phi(x)=a_{c} c n\left(b_{c} x, k\right) \approx \bar{a} \cos (c x)+\overline{\bar{a}} \cos (3 c x)+\ldots
$$


This situation is illustrated by figure 2 .
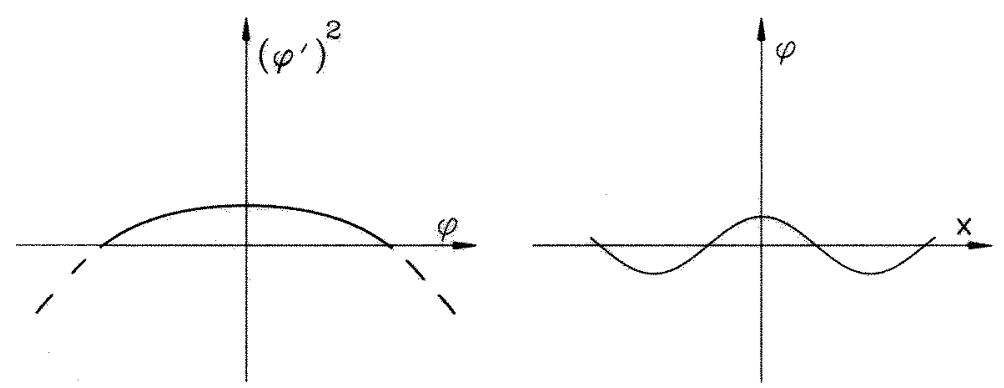

Figure 2.

The change of $a_{c}, b_{c}, k$ values caused by the change of the material constants with temperature, pressure and so on leads to growth of the absolute value of $\phi^{\prime 2}(x)$ maximum at $\phi=0$ point. But this process is not unlimited. It is stopped under $k^{2}=0.5$ and for $0.5<k^{2}<1$ new features in the behaviour of the curve are observed (figure 3 ).
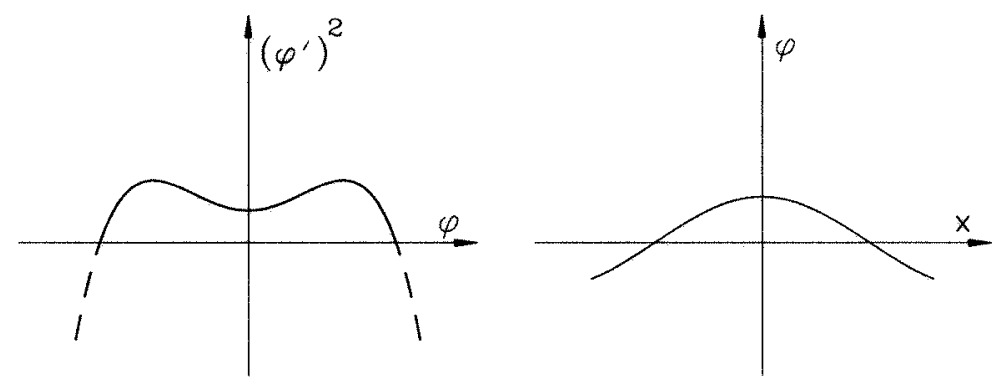

Figure 3.

Spontaneous symmetry breaking ( SSB ) for the discrete group of symmetry - the parity group takes place. Meanwhile $\langle\phi(x)\rangle_{T}=0$. It means that though the "local" order is broken, this symmetry is not broken globally. As for the translational group $x \rightarrow x+C$, the parameter $C$ may take values only proportional to the period $T$ instead of arbitrary ones for the phase 1 .

3. $\phi^{2}(x)$ becomes equal to zero at $\phi=0$ again (see figure 4$)$. In this case $a_{0}=0, a_{1} \neq 0$, and for the model (3) the solution to equation (2) is the soliton :

$$
\phi(x)=a_{c} \operatorname{cn}\left(b_{c} x, 1\right)=\frac{\tilde{a}}{\operatorname{ch}(v x)}=a_{d} d n\left(b_{d} x, 1\right) .
$$

where $d n(x, k)$ is one of the Jacobi elliptic functions. There are neither parity nor translational symmetries in the system. The period $T$ is equal to infinity and $\langle\phi(x)\rangle_{T} \neq 0$, i.e. there is full parity breaking in the system, not only local, but also global. MS2.

The equation $a_{0}=0$. is one of the main characteristics of this PT MS1 $\rightarrow$

Let us evaluate the value of thermodynamical potential for the model (3) in the vicinity of $a_{0} \approx 0$. Using the expansion of the elliptic integrals 
$K(k)$ and $E(k)$ for $k^{2} \approx 1$ [3], we obtain :

$$
\Phi \approx \Phi_{1}+a_{0} \Phi_{2}+a_{0}^{2} \Phi_{3}+a_{0}^{2} \ln \left(\left|a_{0}\right|\right) \Phi_{4},
$$

where $\Phi_{i}, i=1,2,3,4$, are the combinations of $a_{1}$ and $a_{2}$. So, the second derivative of $\Phi$ with respect to $a_{0}$ is proportional to $\ln \left(\left|a_{0}\right|\right)$ whereas $a_{0} \approx 0$.
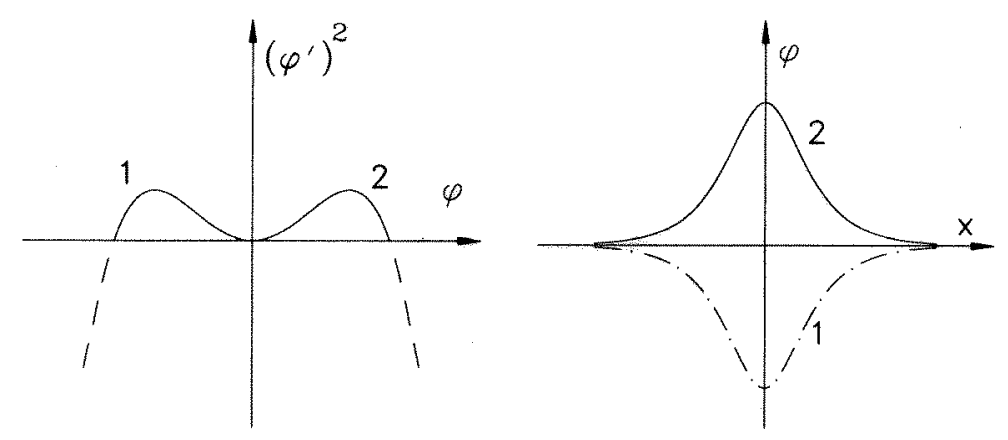

Figure 4.
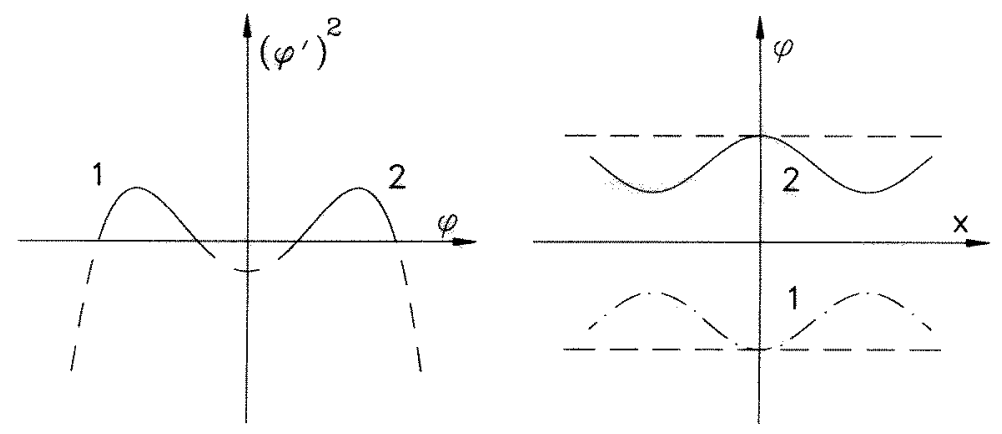

Figure 5.
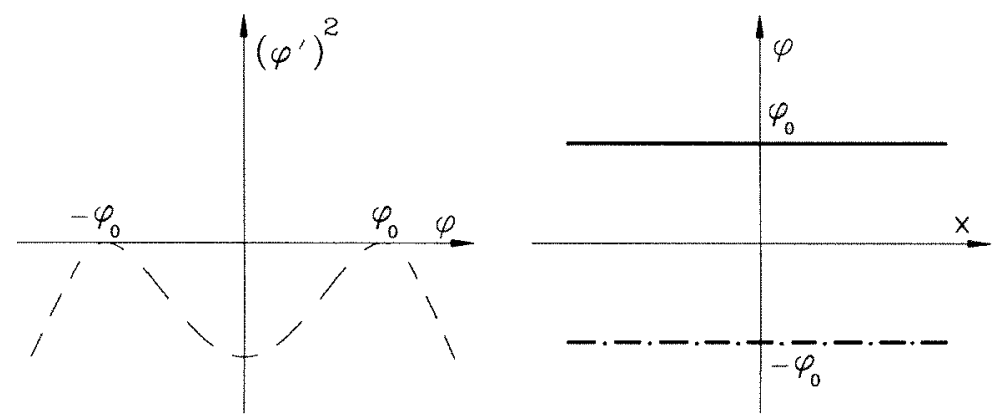

Figure 6 . 
4. MS2 phase - see figure 5. This function is above zero for all the values of $x$ and $\langle\phi(x)\rangle_{T} \neq 0$, the period $T$ is finite. For the model (3) we have

$$
\phi(x)=a_{d} \operatorname{dn}\left(b_{d} x, k\right)
$$

where $0 .<k^{2}<1$. For $k^{2} \approx 1$.

$$
\phi(x)=a_{d} \operatorname{dn}\left(b_{d} x, k\right) \approx \bar{a}(1+\cos (2 \bar{c} x)+\ldots) .
$$

The translational group symmetry $x \rightarrow x+T$ takes place.

5. The final phase $-\phi(x)= \pm \phi_{0}=$ const (see figure 6 ). For the model (3) the point of PT from MS2 into this final phase is characterized by the equation $a_{0}^{2}-4 a_{1} a_{2}=0$.

Translational group symmetry is restored for arbitrary $C: x \rightarrow x+C$.

Thus, one can conclude that the system loses its parity symmetry by parts. And MS may be considered as a very important "tool" of this process. The analysis of PT from the phase $\phi(x)=0$ into the phase $\phi(x)= \pm \phi_{0}$ allows to conclude that modulated structures may be a necessary element of any 2nd-kind PT.

In conclusion consider the following auxiliary potential

$$
\hat{\Phi}=\hat{\Phi}_{0} \int\left[(\Delta f)^{2}+\hat{g} r^{\alpha}(f \vec{\nabla} f)^{2}+\frac{1}{3} r^{2 \alpha} f^{6}\right] d^{d} \vec{r},
$$

where $d$ - is the space dimension, $r=|\vec{r}|$.

For any $d$ the Euler-Poisson equation for the functional (5) possesses symmetry with respect to the dilation group $\overrightarrow{r^{*}}=\lambda \vec{r}, f^{*}\left(\overrightarrow{r^{*}}\right)=\lambda^{-\delta} f(\vec{r})$, where $\delta=(\alpha+2) / 2$. At $\alpha=d-6$ this symmetry becomes variational, i.e. the functional in question acquires it, too. If $f(\vec{r})=f(r)$, then the substitution $t=\ln r, f(r)=r^{-1} \varphi(t)$ transforms the Euler-Poisson equation for (5) to a differential one of (2) type with coefficients depending on $d$ and $\hat{g}$. Here we present such a differential equation for $d=6$ ( i.e. $\alpha=0)$ :

$$
\varphi^{(I V)}-\hat{g}\left(\varphi^{2} \varphi^{\prime \prime}+\varphi \varphi^{\prime 2}\right)-10 \varphi^{\prime \prime}+9 \varphi+2 \hat{g} \varphi^{3}+\varphi^{5}=0 .
$$

An exact solitonic solution to (4) type results from the existence of a hidden $d$-dimensional variational symmetry of the differential equation (6).

\section{References}

[1] Klepikov V.F. Modulated structures and phase transitions. // Sov. J. Low Temp. Phys., 1991, vol. 17, No 9, p. 1166-1169.

[2] Klepikov V.F. Modulated structures of one-component order parameter. // J. Phys. C8 (Paris), 1988, vol. 49, No 12, p. 1805-1806.

[3] Jahnke E., Emde F. Tables of functions. New-York, Dover Publications, 1945. 


\title{
МОДУЛЬОВАНІ СТРУКТУРИ ПАРАМЕТРА ПОРЯДКУ ТА СПОНТАННЕ ПОРУШЕННЯ ПАРНОСТІ
}

\author{
В.Ф.Клепіков, С.В.Березовський
}

Модульовані структури параметра порядку розглядаються як важливий необхідний елемент фазових переходів. Стійкі модульовані структури є результатом конкуренції між деякими високоградієнтними доданками. Існує дві фази модульованих структур, що мають різні рівні спонтанного порушення симетрії для групи парності. При точці переходу між цими фазами просторовий розподіл параметра порядку є таким самим як для bell-солітона. Оцінюється термодинамічний потенціал в околі цієї точки. 